\title{
Experimental Characterization of the Viscoplastic Material Behaviour of Thermosets and Thermoplastics
}

\author{
Markus Kästner, Swen Blobel, Martin Obst, Karin Thielsch and Volker Ulbricht \\ Technische Universität Dresden, Institute of Solid Mechanics, D-01062 Dresden, Germany \\ $\{$ Markus.Kaestner, Swen.Blobel, Martin.Obst, Karin.Thielsch, \\ Volker.Ulbricht\}@tu-dresden.de
}

\section{Keywords: Polymer, Composite, Viscoplasticity, Relaxation}

\begin{abstract}
In this contribution the mechanical behaviour of polymeric matrix materials is analysed for both thermoplastics (Polypropylene) and thermosets (RTM6, RIM935). The results obtained from tensile tests carried out at different velocities indicate a nonlinear, inelastic material behaviour with strainrate dependence. For the clear identification and quantification of the nonlinearities, the experimental procedure has been extended to relaxation experiments and deformation controlled loading-unloadingprocesses with intermediate relaxations. Based on the experimental observations a small-strain viscoplastic material model is derived and material parameters are identified. The stress-strain-curves computed for different load histories are compared to the experimental results.
\end{abstract}

\section{Introduction}

The authors have applied multi-scale modelling and simulation techniques [1] to predict the effective linear elastic stiffness properties as well as the macroscopically nonlinear material behaviour for textilereinforced polymers using only the properties of the individual constitutents and their geometrical arrangement in the composite. The observed macroscopic nonlinearities are, amongst others, driven by damage effects and the inelastic material behaviour of the polymeric matrices. Typical thermoplastics and thermosets often show strong strain-rate dependence in combination with irreversible deformation. Here the nonlinear material behaviour of different matrices is analysed experimentally and modelled numerically.

\section{Classification of Material Behaviour}

Concerning the classification of the observed phenomena we follow a proposal of Haupt [2] who suggests a classification based on the strain-rate dependence of the material behaviour and on the examination of an equilibrium relation. Examples for equilibrium points are the terminal points observed in relaxation $(\dot{\varepsilon}=0)$ or creep $(\dot{\sigma}=0)$ experiments. A set of equilibrium points for different strain states forms the equilibrium relation $\sigma^{\text {eq }}$.

If the stress-strain-curves obtained from experiments carried out at various strain-rates differ from each other, the material behaviour is named strain-rate dependent. The difference between the stress-strainrelation measured in an experiment at finite strain-rate $|\dot{\varepsilon}|>0$ and the equilibrium relation is called overstress $\sigma^{\text {ov }}$. The second essential feature of the material behaviour is the shape of the equilibrium relation obtained in loading and unloading processes. The classification distinguishes between equilibrium relations with and without hysteresis (Fig. 1).

In the case of strain-rate independent material behaviour every stress-strain-curve is an equilibrium relation. In the case of strain-rate dependence the existence of an hysteresis in the stress-strain-curve 


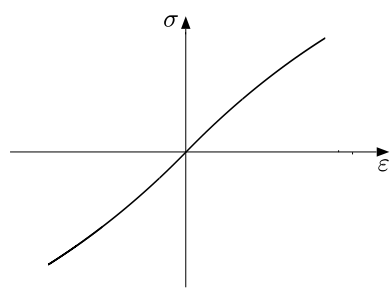

(a) Elasticity

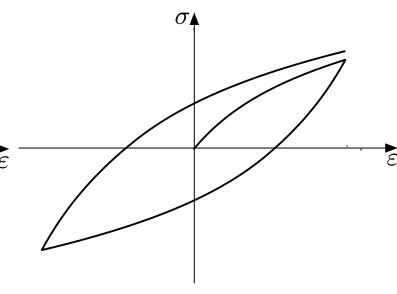

(b) Plasticity

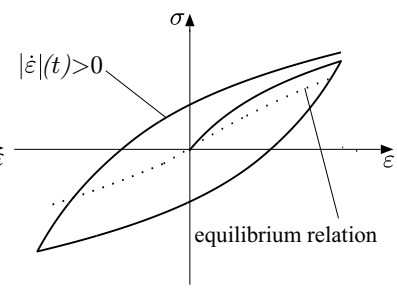

(c) Viscoelasticity

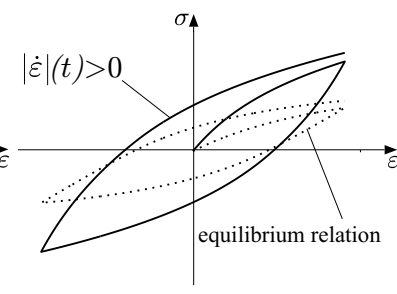

(d) Viscoplasticity

Figure 1: Four types of material behaviour

does not allow for a clear classification of the material behaviour. Therefore, strain-rate dependent and independent phenomena have to be separated by a suitable experimental procedure and the shape of the equilibrium relation has to be determined. According to this, the following classification can be made:

- Elasticity: strain-rate independent without hysteresis (Fig. 1(a)),

- Plasticity: strain-rate independent with hysteresis (Fig. 1(b)),

- Viscoelasticity: strain-rate dependent without equilibrium hysteresis (Fig. 1(c)),

- Viscoplasticity: strain-rate dependent with equilibrium hysteresis (Fig. 1(d)).

\section{Testing Procedure}

The testing procedure applied is chosen in accordance with the proposed classification of the material behaviour. All displacement controlled experiments are carried out on a standard servo-hydraulic testing machine with a maximum force of $16 \mathrm{kN}$. The strain-state in the specimen is measured by a laser extensometer and digital image correlation (ARAMIS). An air conditioning system provides approximately constant ambient temperatures of $\vartheta=296 \mathrm{~K}$.

In the first instance, monotonic tensile tests at constant strain-rates (Fig. 2(a)) are performed. Any

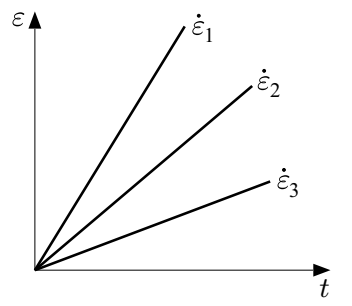

(a) Monotonic tensile tests

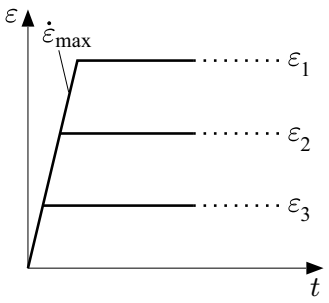

(b) Relaxation tests

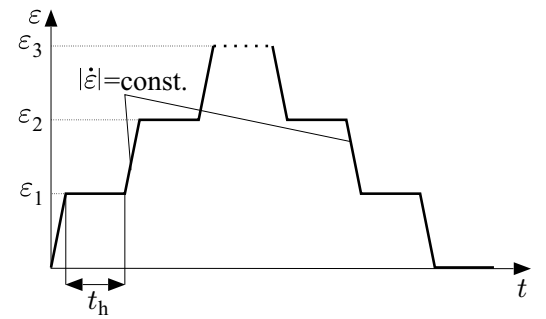

(c) Loading and unloading tests with intermediate relaxations

Figure 2: Testing procedure

differences between the stress-strain-curves obtained for different strain rates $\dot{\varepsilon}_{1}>\dot{\varepsilon}_{2}>\dot{\varepsilon}_{3}$ indicate the strain-rate dependence of the material behaviour. The quantification of the strain-rate dependence is then accomplished by relaxation tests at different strain levels $\varepsilon_{1}>\varepsilon_{2}>\varepsilon_{3}$ (Fig. 2(b)). 
The results obtained in tensile tests and relaxation experiments do not allow for an unambiguous classification as no unloading processes are considered. Hence, no information on the existence of an hysteresis exists. The testing procedure is therefore extended by loading and unloading processes with intermediate holding times at discrete strain levels in order to separate strain-rate dependent and independent effects (Fig. 2(c)).

\section{Material Behaviour of Polypropylene}

As one example for the proposed classification and characterization procedure, the material behaviour of Polypropylene has been investigated experimentally. In order to compare the material behaviour in monotonic tensile tests carried out at different constant velocities according to Fig. 2(a)) three engineering stress-strain-curves for distinct velocities of 1, 10 and $100 \frac{\mathrm{mm}}{\mathrm{min}}$ are shown in Fig. 3.

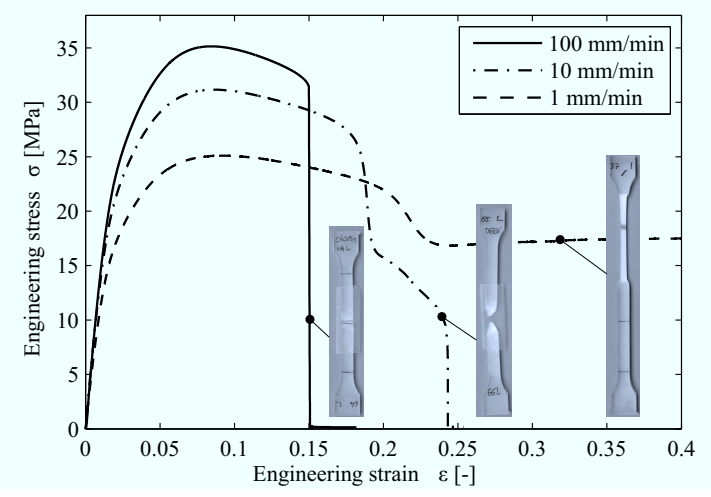

(a) Stress-strain-curves and deformed specimen

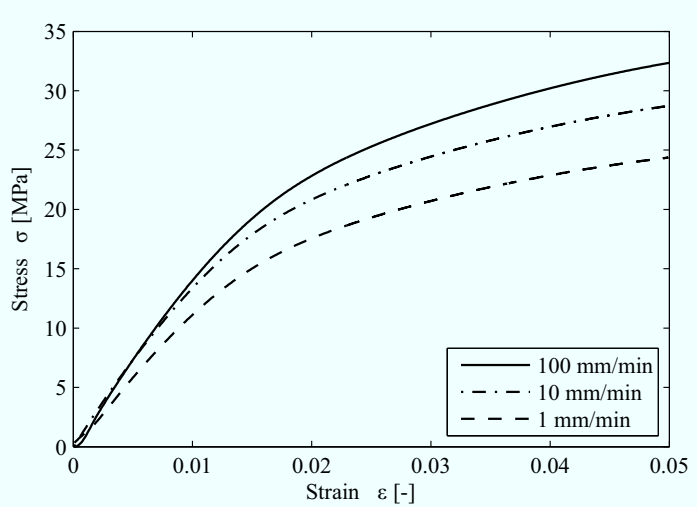

(b) Stress-strain-curves for $0 \leq \varepsilon \leq 0.05$

Figure 3: Comparison of characteristic stress-strain-curves for three distinct velocities

With higher velocities a clear nonproportional increase in the stress level and the stiffness can be observed. Simultaneously, the strain to failure and the amount of inelastic deformation decrease. At a velocity of $100 \frac{\mathrm{mm}}{\mathrm{min}}$ the specimen fails at a maximum strain of 15 per cent. In contrast to this, no failure can be observed at a speed of $1 \frac{\mathrm{mm}}{\mathrm{min}}$. In this case a neck develops and propagates through the specimen at a virtually constant load level. During this process the inelastic deformation is concentrated in two process zones at both ends of the neck.

Relaxation experiments allow for the separation of the strain-rate dependent and independent fractions of stress and are therefore of great importance for the characterization of the strain-rate dependent material behaviour. Fig. 4(a) shows the stress-time-curve of a relaxation test with a holding $t_{\mathrm{h}}$ of 48 hours. After a pronounced relaxation of stress at the beginning, the time-rate of stress decreases until the relaxation stopps after approximately 48 hours. With respect to the proposed material model this stress state is assumed to be a state of equilibrium.

So far the strain-rate dependence of the material behaviour has been demonstrated in tensile tests, relaxation experiments allow for its quantification. However, for the clear classification of the material behaviour the shape of the equilibrium relation has to be assessed. Suitable experiments are loading and unloading processes with intermediate holding times (Fig. 2(c)). For practical reasons holding times of 48 hours and more are not reasonable in these experiments. To this, a reduced holding time for intermediate relaxations $\Delta \sigma_{\mathrm{r}}\left(t_{\mathrm{h}_{\mathrm{ir}}}\right)=0.9 \cdot \Delta \sigma_{\mathrm{r}}\left(t_{\mathrm{h}}=48 \mathrm{~h}\right)$ is defined, that is 90 percent of the overstress relaxes during $t_{\mathrm{h}_{\mathrm{i}}}$. For Polypropylene the condition above leads to holding times of $t_{\mathrm{h}_{\mathrm{ir}}} \approx 3 \mathrm{~h}$. 


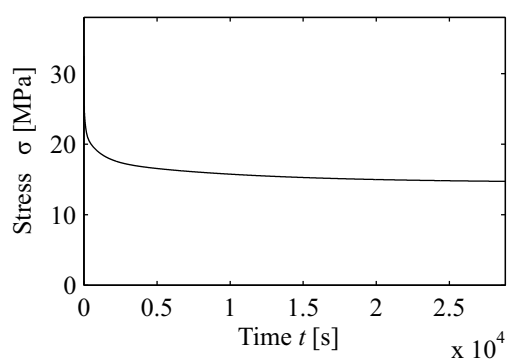

(a) Stress-time-curve for relaxation experiment

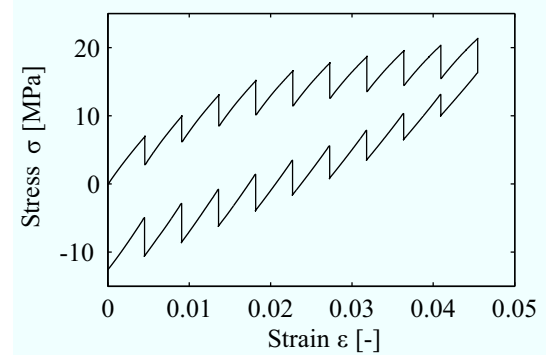

(b) Stress-strain-curve for loading and unloading with intermediate relaxations

Figure 4: Results for relaxation experiments and loading-unloading processes with intermediate holding times

All loading and unloading processes are carried out at a velocity of $10 \frac{\mathrm{mm}}{\mathrm{min}}$ and discrete points of the equilibrium relation are determined by intermediate relaxation processes every $\Delta \varepsilon=0.045$. Fig. 4(b) shows the resulting stress-strain-curves for this experiment. Although the relaxation of the overstress is not completely finished after the reduced holding time of 3 hours, the experiment indicates an equilibrium hysteresis as the terminal points at a certain strain level do not coincide for the loading and unloading path. At least the existence of an equilibrium relation is proven by the experiment.

\section{Material Behaviour of Thermosets}

Compared to the highly inelastic behaviour of Polypropylene, the two considered thermosets - RIM935 and RTM6 - show only limited inelastic deformation. In monotonic tensile tests (Fig. 5(a), Fig. 5(c)) a minor strain-rate dependence can be detected. The strain to failure is considerably lower than for Polypropylene. This behaviour is on the one hand due to the different microstructures of thermosets and thermoplastics as well as due to the fact that both thermosets possess glass transition temperatures $\vartheta_{\mathrm{g}}>373 \mathrm{~K}$. In contrast to Polypropylene $\left(\vartheta_{\mathrm{g}}=273 \mathrm{~K}\right)$ RIM935 and RTM6 are tested below their glass transition temperatures. In order to quantify the strain-rate dependence and to find out about the amount of stress relaxation which can be of interest for manufacturers of fibre-reinforced polymers relaxation experiments (Fig. 5(b), Fig. 5(d)) have been performed. The procedure is currently extended to different temperature levels below and above the glass transition temperatures of the considered thermosets.

\section{A Material Model for Polymers}

In literature there are various approaches to the simulation of the mechanical behaviour of polymers (e.g. $[3,4,5])$. Here, in line with the presented experimental procedure, we propose constitutive equations for modelling the inelastic material behaviour of polymers which can be characterized as a viscoplastic material model based on an overstress formulation. The stress $\sigma=\sigma^{\mathrm{eq}}+\sigma^{\mathrm{ov}}$ is a combination of strain-rate independent equilibrium stress $\sigma^{\text {eq }}$ and strain-rate dependent overstress $\sigma^{\text {ov }}$. Together with the experiments performed, this allows for a clear identification of the necessary material parameter. A similar approach is presented in [6].

The material model for the equilibrium stress consists of the combination $\sigma^{\text {eq }}=\sigma^{\text {eq,e }}+\sigma^{\text {eq,end }}$ of a 


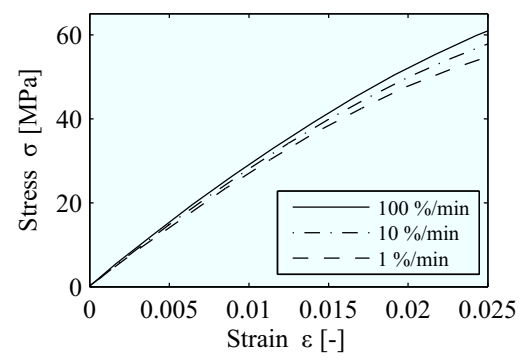

(a) RIM935: Tensile tests

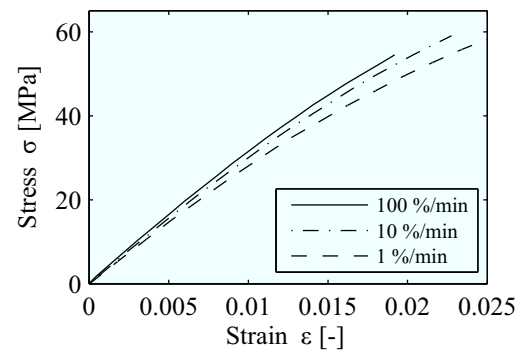

(c) RTM6: Tensile tests

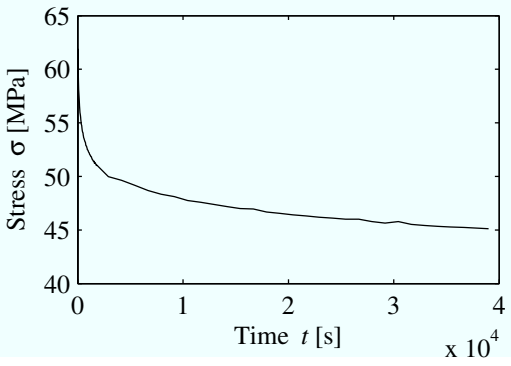

(b) RIM935: Relaxation experiment

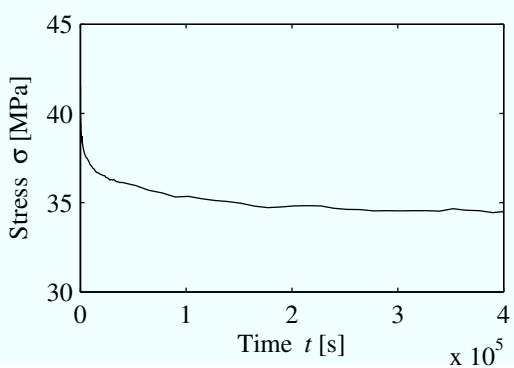

(d) RTM6: Relaxation experiment

Figure 5: Experimental results obtained for two thermosets - RIM935 and RTM6

nonlinear elastic stress-strain-relation $\sigma^{\mathrm{eq}, \mathrm{e}}$ and an endochronic model [7, 8] of plasticity $\sigma^{\mathrm{eq}, \text { end }}$

$$
\begin{aligned}
\sigma^{\text {eq,e }} & =\frac{E_{1}}{1+\alpha|\varepsilon|} \varepsilon \\
\sigma^{\text {eq,end }} & =E_{2}\left(\varepsilon-q^{\text {end }}\right) \quad \text { with } \quad q^{\text {end }}(z)=\beta\left(\varepsilon(z)-q^{\text {end }}(z)\right) \quad \text { and } \quad \dot{z}(t)=|\dot{\varepsilon}|
\end{aligned}
$$

where $q^{\text {end }}$ is an internal variable used to model the hysteresis in the equilibrium relation. Strainrate independence is ensured by the use of a generalized arclength $z$. If no equilibrium hysteresis is observed, only the elastic part of the stress-strain-relation can be used. Linear elastic material behaviour is included in the model for $\alpha=0$. The material properties are identified from fitting the model prediction to the terminal points of the intermediate relaxations.

The constitutive formulation for overstress is a generalized MAXWELL-model with $n_{\mathrm{v}}$ parallel elements

$$
\begin{aligned}
\sigma^{\mathrm{ov}} & =\sum_{i=1}^{n_{\mathrm{v}}} \sigma_{i}^{\mathrm{ov}} \\
\sigma_{i}^{\mathrm{ov}} & =c_{i}\left(\varepsilon-q_{i}^{\mathrm{ov}}\right) \quad \text { with } \quad \dot{q}_{i}^{\mathrm{ov}}=\frac{1}{\tau_{i}}\left(\varepsilon-q_{i}^{\mathrm{ov}}\right)=\frac{c_{i}}{\eta_{i}}\left(\varepsilon-q_{i}^{\mathrm{ov}}\right) .
\end{aligned}
$$

The parameters of the model $c_{i}$ and $\tau_{i}$ form a discrete relaxation spectrum which can be determined from relaxation experiments using the window algorithm of EMRI and TSCHOEGL [9].

Nonlinear viscoelastic behaviour, which can be observed for many polymers, requires an extension of the linear overstress model. A nonlinear strain-rate dependence can be introduced by replacing the constant viscosity $\eta_{i}$ with a function $\tilde{\eta}_{i}=\eta_{i} \exp \left(-\frac{\left|\sigma^{\mathrm{ov}}\right|_{0}}{s_{0}}\right)$ depending on the current overstress.

The proposed material model was applied to simulate the mechanical behaviour of three different polymers - the thermoplastic Polypropylen and the thermosets RIM935 and RTM6. After the identification of the necessary material parameters the results illustrated in Fig. 6 have been obtained. 


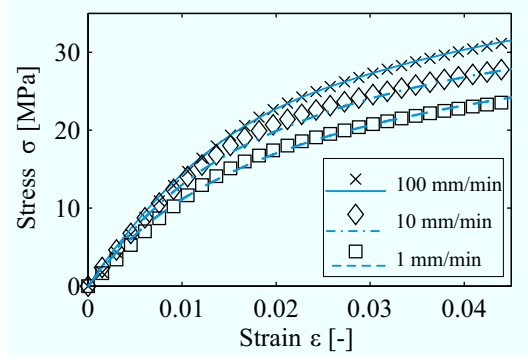

(a) Polypropylene: Tensile tests

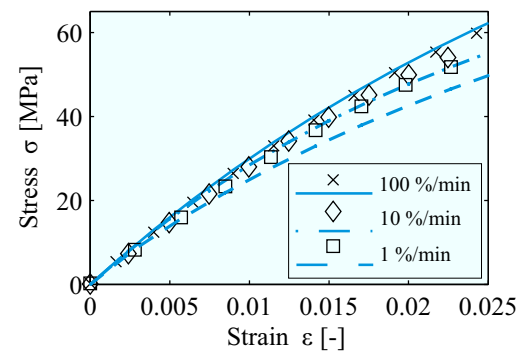

(c) RIM935: Tensile tests

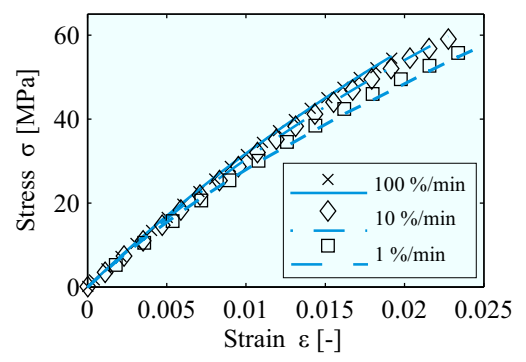

(e) RTM6: Tensile tests

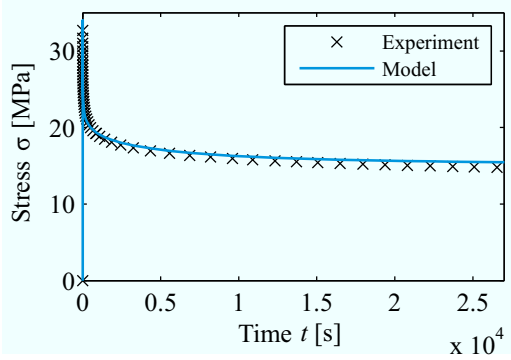

(b) Polypropylene: Relaxation experiment

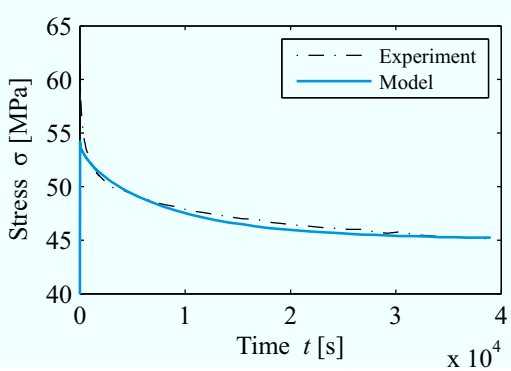

(d) RIM935: Relaxation experiment

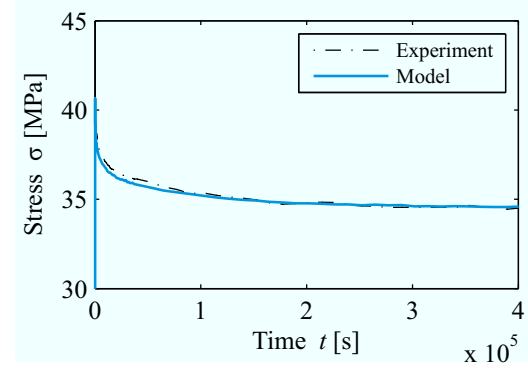

(f) RTM6: Relaxation experiment

Figure 6: Comparison of experimental and numerical results obtained for three polymers

\section{References}

[1] M. Kästner, G. Haasemann, J. Brummund, V. Ulbricht, in: Mechanical response of composites, edited by P.P. Camanho, C.G. Davila, S.T. Pinho, J.J.C. Remmers, Computational methods in applied sciences (Springer, 2008).

[2] P. Haupt, Acta Mechanica 100 (1993), p. 129 - 154.

[3] A. Lion, Continuum Mechanics and Thermodynamics 8 (1996), p. 153 - 169.

[4] E. Krempl, F. Khan, International Journal of Plasticity 19 (2003), p. 1069 - 1095.

[5] A. D. Drozdov, J. d.C. Christiansen, Polymer 48 (2007), p. 3003 - 3012.

[6] S. Hartmann, Arch. Appl. Mech. 76 (2006), p. 349 - 366.

[7] K. Valanis, Archives of Mechanics 23 (1971), p. 517 - 533.

[8] T. Kletschkowski, U. Schomburg, A. Bertram, Mechanics of Time-Dependent Materials 8 (2004), p. $119-135$.

[9] I. Emri, N.W.Tschoegl, Rheologica Acta 32 (1993), p. 311 - 321. 